\title{
Prediction of Refractive Outcome of Second Eye with Partial Adjustment of Intraocular Lens Power of the First Eye
}

\author{
Nooshin Bazzazi ${ }^{1}$, Pouyan Pahlevani², Mehdi Alizadeh ${ }^{3, *}$ \\ ${ }^{I}$ Associate Professor, Department of Ophthalmology, School of Medicine, Hamadan University of Medical Sciences, \\ Hamadan, Iran \\ ${ }^{2}$ Resident, Department of Ophthalmology, Imam Hossein Hospital, Shahid Beheshti University of Medical Sciences, Tehran, \\ Iran \\ ${ }^{3}$ Assistant Professor, Department of Ophthalmology, School of Medicine, Hamadan University of Medical Sciences, \\ Hamadan, Iran \\ * Corresponding Author: Mehdi Alizadeh, Department of Ophthalmology, School of Medicine, Hamadan University of \\ Medical Sciences, Hamadan, Iran.Email: mahaliz@yahoo.com
}

Received: 29.07 .2019

Accepted: 17.11.2019

How to Cite this Article: Bazzazi N, Pahlevani P, Alizadeh M. Prediction of Refractive Outcome of Second Eye with Partial Adjustment of Intraocular Lens Power of the First Eye. Avicenna J Clin Med. 2019; 26(3): 137-142. DOI: $10.29252 /$ ajcm.26.3.137

\section{Abstract}

Background and Objective: This study aimed at comparing the results of refractive cataract surgery in patients undergoing sequential surgeries in both eyes. Considering the preoperative refraction and final refraction of the first eye, it is possible to correct the refrative result of cataract surgery using the adjustment of the second eye intraocular lens (IOL) power.

Materials and Methods: This study was conducted based on a matchedpair clinical-trial design. Regarding the final result of the first eye refraction, the adjustment of the second eye IOL power was determined by $50 \%$ of uncorrected refractive error. Finally, the mean spherical equivalent (SE) of the first and second eyes were determined, and the differences were analyzed using a paired t-test at a significant level of $95 \%$.

Results: A total of 470 candidates who underwent bilateral sequential cataract surgery were investigated in this study. The refractive errors ranged from -8.25 to +6.50 and from -6.75 to +7.25 for the first and second eyes, respectively. The mean values of SE for the first and second eyes were 2.55 and 2.48 preoperatively, and 1.13 and 0.47 postoperatively, respectively. Moreover, there was a significant difference between the two groups $(\mathrm{P}<0.001)$.

Conclusion: The adjustment of IOL power for the second eye by $50 \%$ of uncorrected refractive error of the first eye led to acceptable outcomes.

Keywords: Cataract, Intraocular Lens Power, Refractive Outcome 
dof: $10.29252 / \mathrm{ajcm} .26 .3 .137$

\title{
بررسى بيامد رفر كتيو جراحى كاتاراكت در جشم دوم با تنظيم نسبى قدرت لنز داخل جشمى در جشم اول
}

\author{
نوشين بزازى'، يو يان بههلوانى'، مهدى عليزاده

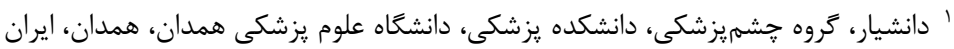

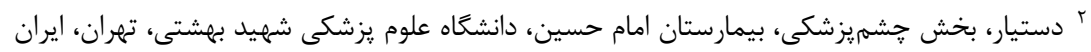

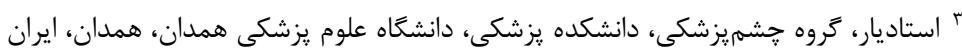
mahaliz@yahoo.com : نويسنده مسئول: مهدى عليزاده، كروه حشمريزشكى، دانشكده يزشكى، دانشًاه علوم يزشكى همدان، همدان، ايران. ايميل

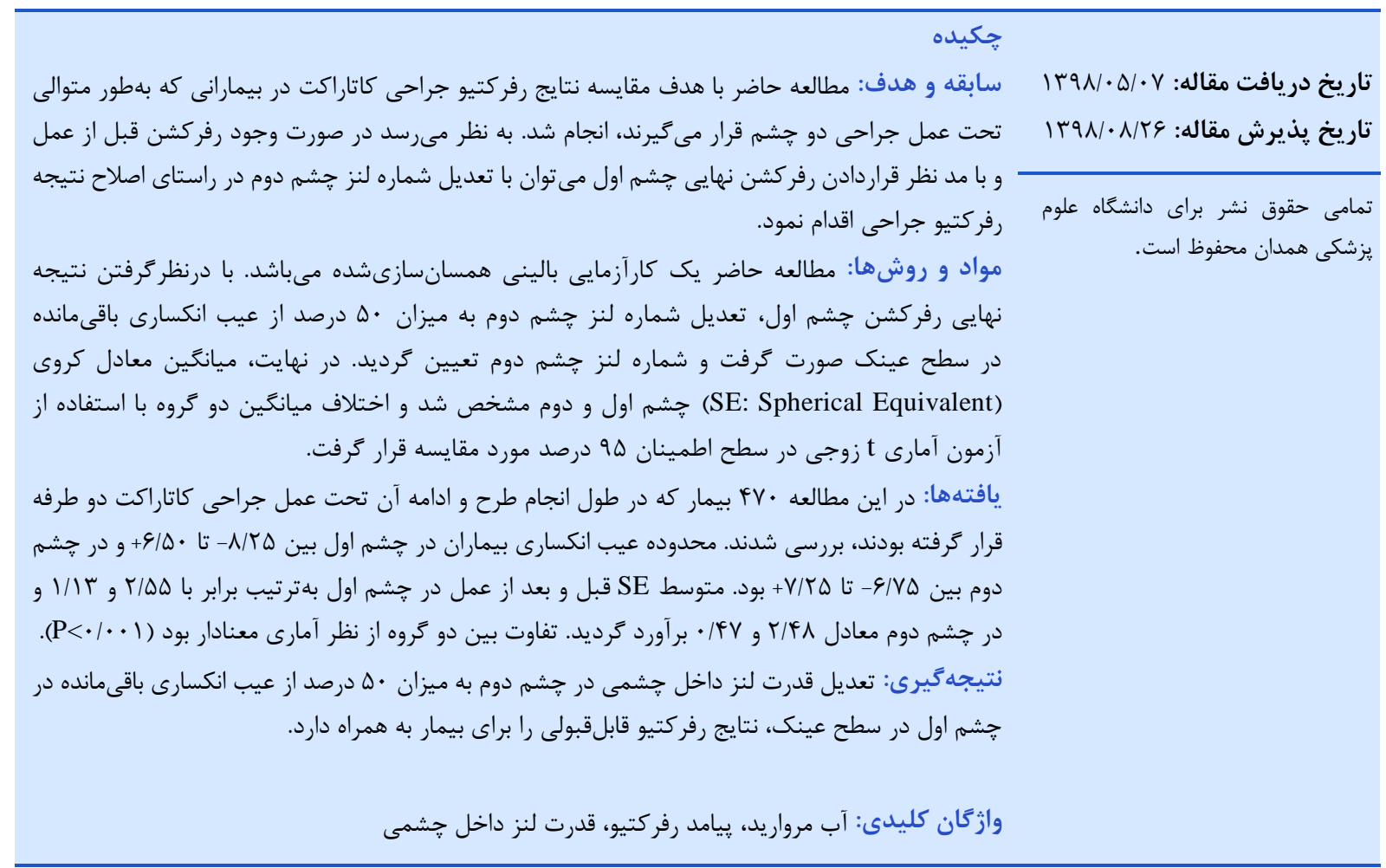

قدرت لنز داخل جشمى كه از مهمترين مراحل در آمادهسازى

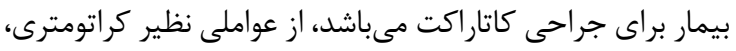

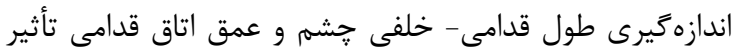

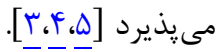

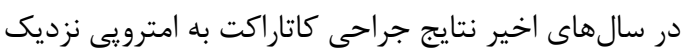

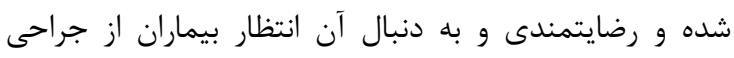

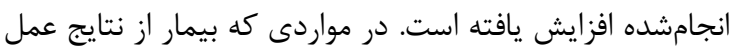
رضايت ندارد، يك علت مههم آن محاسبه غيردقيق شماره لنز

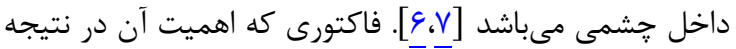

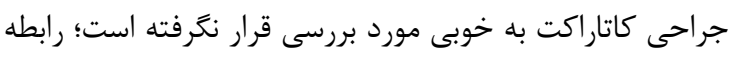

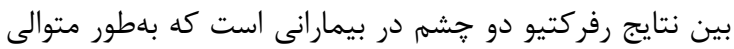

كاتاراكت مهمترين علت كورى قابلبرگشت است و جراحى كاتاراكت از متداولترين و موفقترين اعمال جراحى درين در حوزي

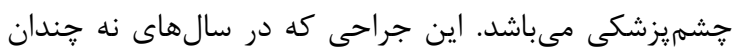

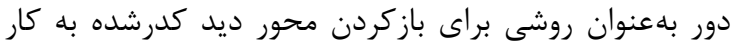

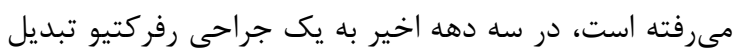

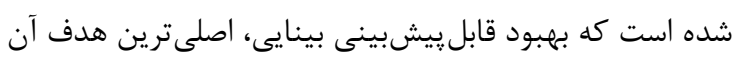

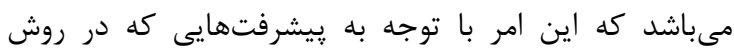

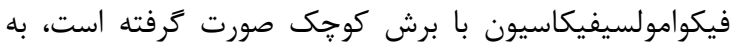

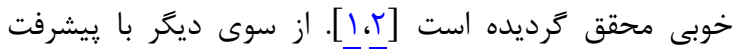

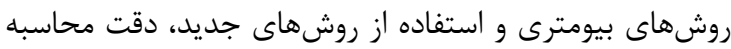
(Intraocular Lens) IOL 
حذف شدند.

در اين مطالعه اندازهگيرى قدرت لنز داخل جشمى باند إنا

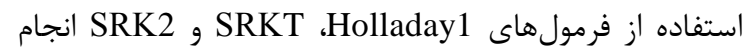
شد و براى دقت بيشتر، بيماران از نظر AL در جهار گروه جاى كرفتند.

Holladay1, SRK T: AL $<Y T$

SRKT, SRK2: AL Yr-YY/Fq

SRKT, SRK2: AL $r F / Q \cdot-r \&$

SRKT, SRK2: AL>Y\&

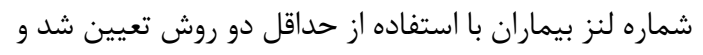

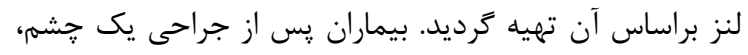

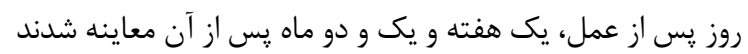
و نتايج رفركتيو جراحى آنها ثبت كرديد.

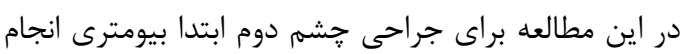

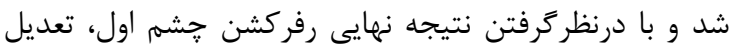

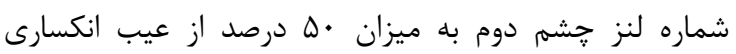

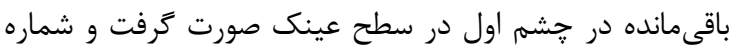

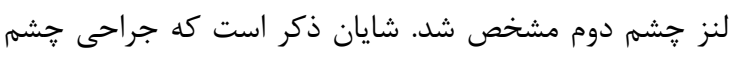

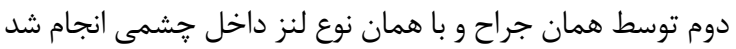

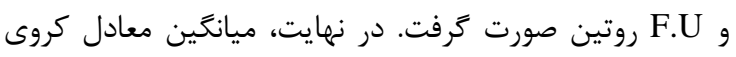

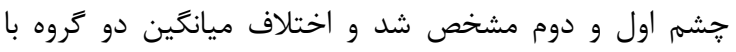

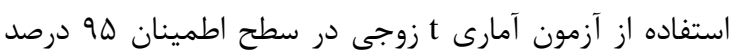

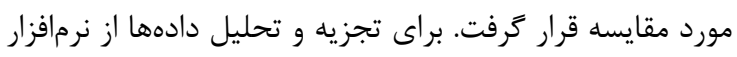

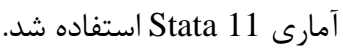

\section{يافتهها}

در مطالعه حاضر •VV ب بيمار مبتلا به كاتاراكت دو طرفه كه

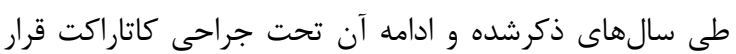

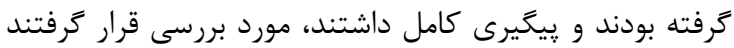

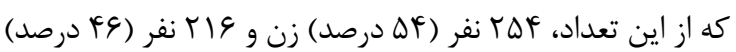

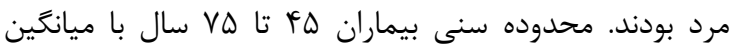

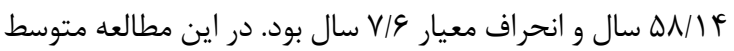

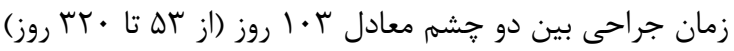

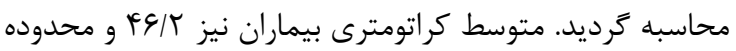

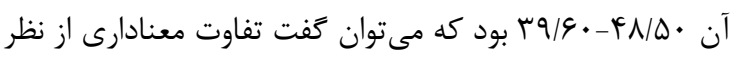

$$
\text { آمارى بين دو جشم وجود ندارد. }
$$

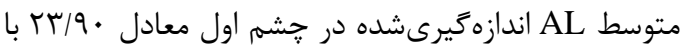

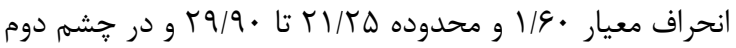

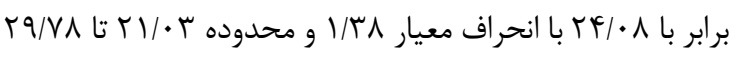

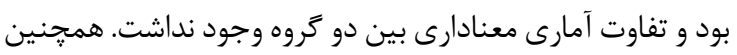

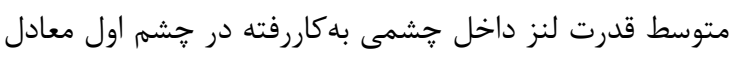

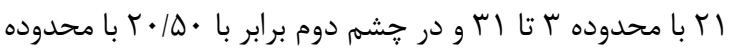

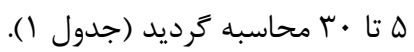
متوسط SE نيز قبل و بعد از عمل در حشمه اول معاد معادل
تحت عمل جراحى كاتاراكت قرار مى ميرند. به نظر مىرسد در

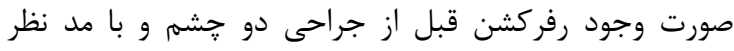

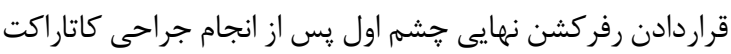

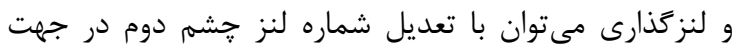

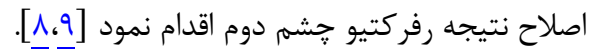

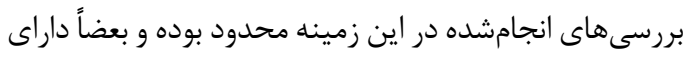

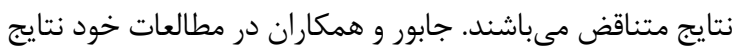

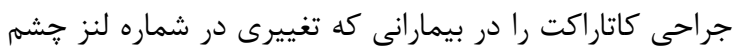

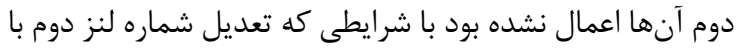

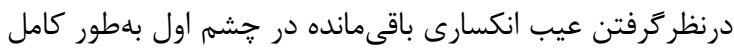

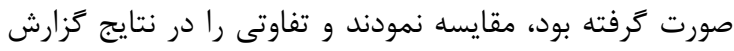

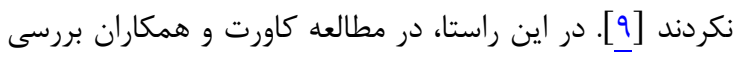

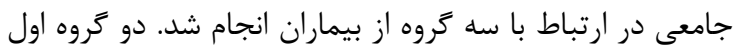

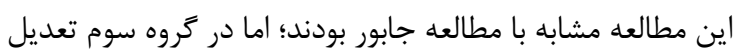

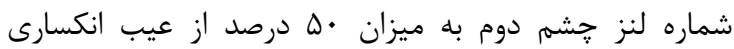

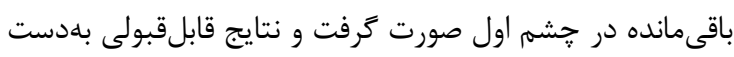

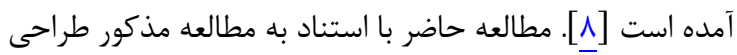

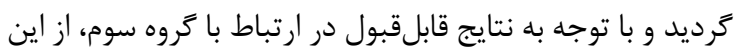

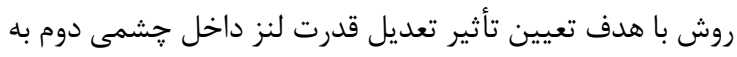

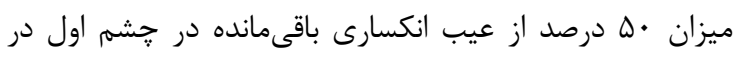

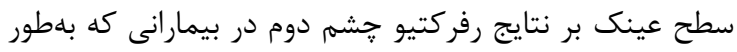

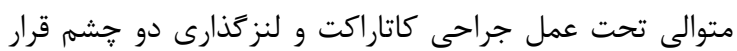
گرفته بودند، استفاده شد.

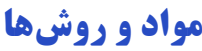

مطالعه حاضر يك كارآزمايى بالينى همسانسازىشده

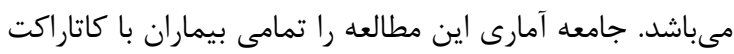

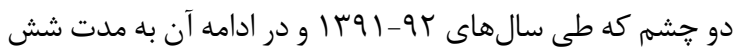

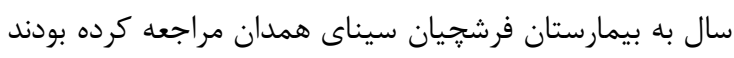

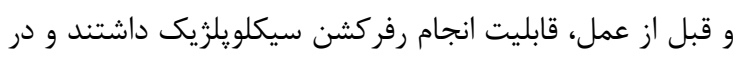

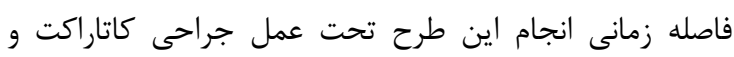

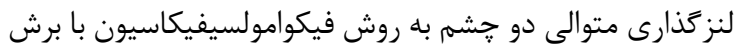

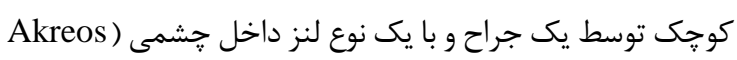
(AO60 Bausch \&Lomb قرار ترفتند، تشكيل دادند.

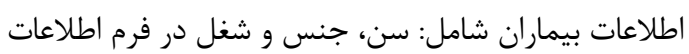

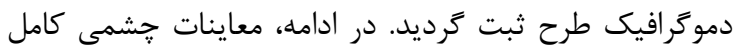

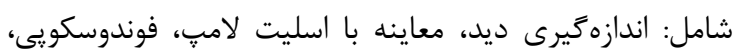

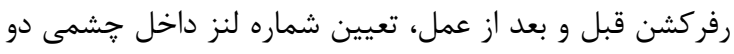
جشم (اندازميرى طول اتز يال و كراتومترى با باستفاده از دستخاه

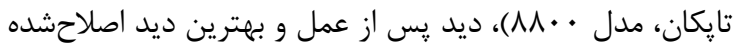

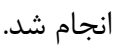

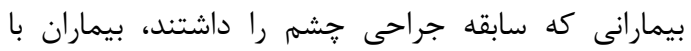

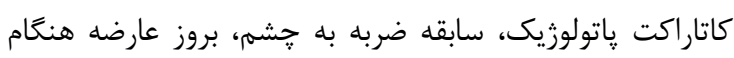

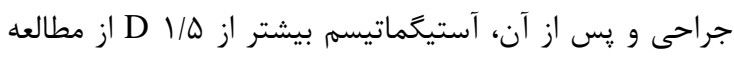




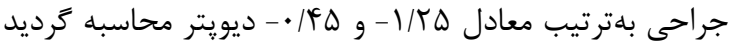
كه اين تفاوت از نظر آمارى معنادار بود (شكل (1).

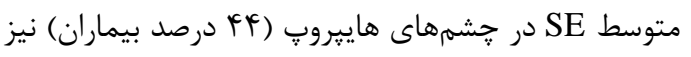

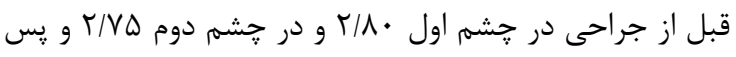

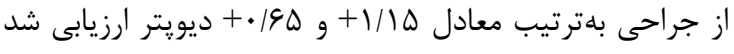

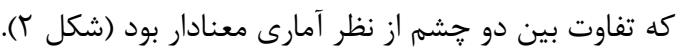

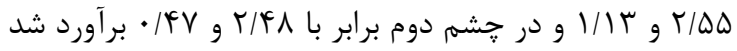

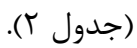

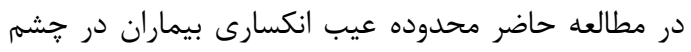

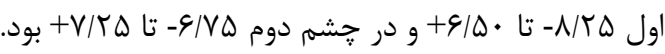

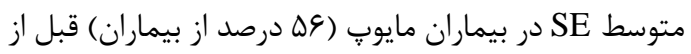

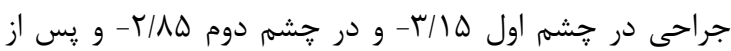

جدول ا: اطلاعات بيومتريك جشم اول و دوم در بيمار ان مورد مطالعه

\begin{tabular}{|c|c|c|c|c|}
\hline IOL POWER & $\mathbf{K R}$ & $\mathbf{A L}$ & & \\
\hline $\begin{array}{c}r \\
r-r\end{array}$ & $\begin{array}{c}r \Delta / \Lambda \\
F \wedge / \Delta \cdot-\Delta \cdot / r q\end{array}$ & $\begin{array}{l}r r / q \cdot \pm 1 / 9 . \\
r 1 / r \Delta-r q / q .\end{array}$ & محدوده & جشم اول \\
\hline $\begin{array}{l}r \cdot / \Delta \cdot \\
\Delta-r \cdot\end{array}$ & $\begin{array}{c}r \varepsilon / l \\
r q-F \wedge / v \Delta\end{array}$ & $\begin{array}{l}r \Psi / \cdot \Lambda \pm I / r \Lambda \\
r I / r-r q / Y \Lambda\end{array}$ & محدوده & جشم دوم \\
\hline
\end{tabular}

جدول ז: رفركشن جشم اول و دوم در بيماران مورد مطالعه قبل و بعد از جراحى

\begin{tabular}{|c|c|c|c|c|c|}
\hline \multirow[b]{2}{*}{ سطح معنادارى } & \multicolumn{2}{|c|}{ بعد از عمل } & \multicolumn{2}{|c|}{ قبل از عمل } & \\
\hline & انحراف معيار & ميانتين & انحراف معيار & ميانَين & \\
\hline$\cdot 1 \cdot \cdot 1$ & .199 & 1/11 & $r / 9 V$ & $r / \Delta \Delta$ & جشم اول \\
\hline$\cdot 1 \cdots 1$ & $\cdot / \Delta V$ & $\cdot / F V$ & $1 / V \Delta$ & $T / F \Lambda$ & جشم دوم \\
\hline
\end{tabular}

\section{Refraction in Myopic eyes}

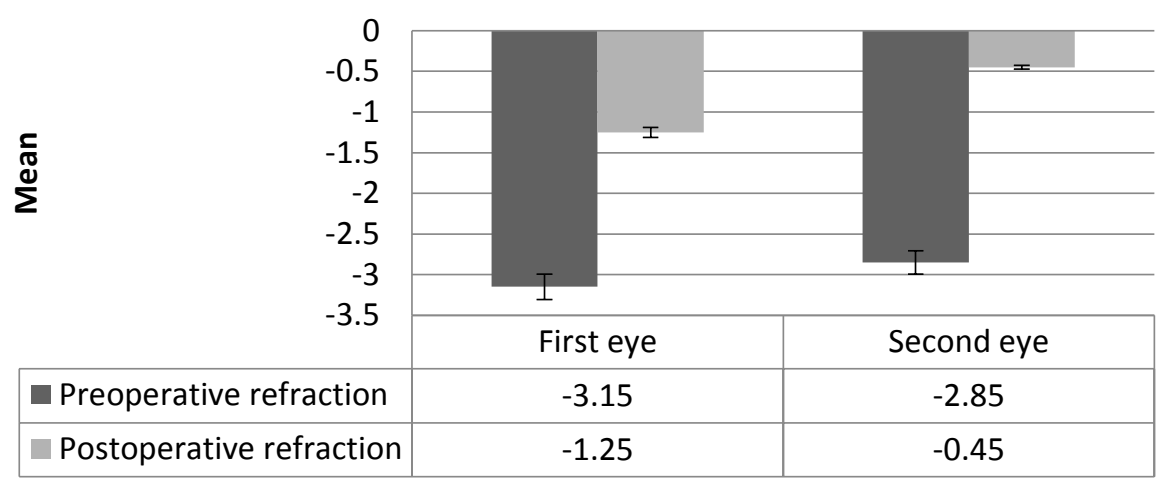

شكل ا: رفركشن جشم اول و دوم در بيماران مايوٍ قبل و بعد از جراحى

\section{Refraction in Hyperopic eyes}

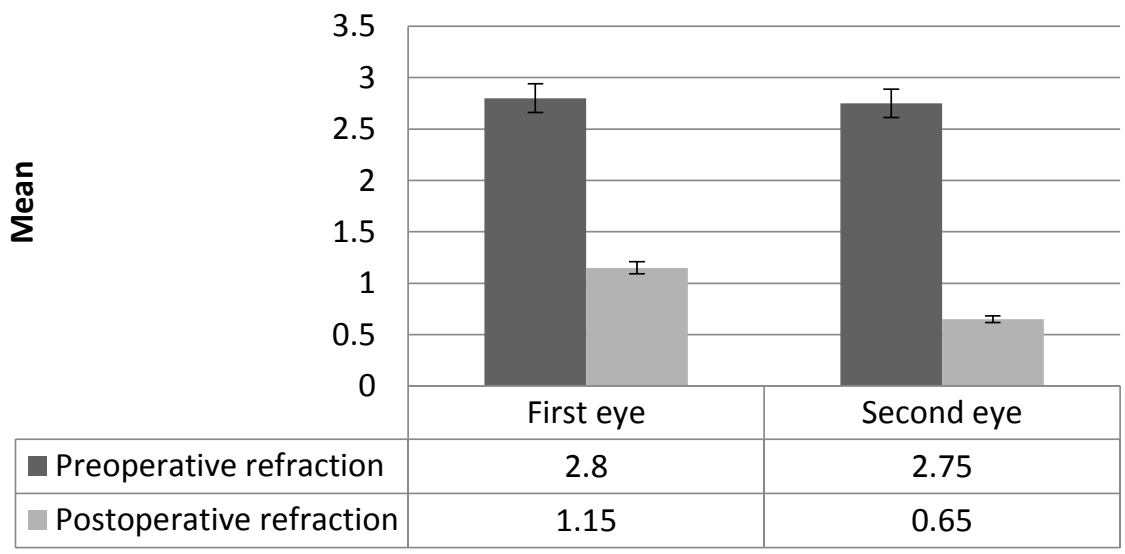

شكل ז': رفر كشن جشم اول و دوم در بيماران هايِروٍ قبل و بعد از جراحى 
كزارش كرديد [1]].

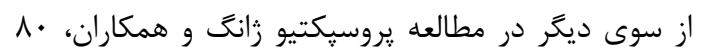

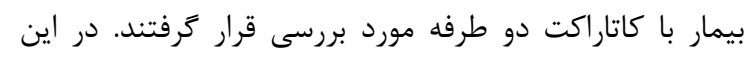

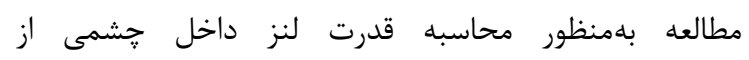

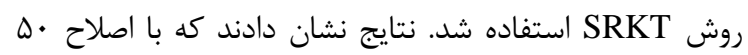

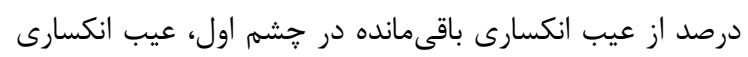

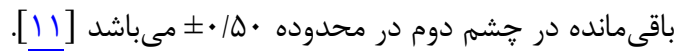

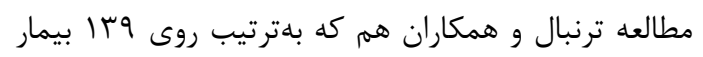
در استراليا و هـ9 بيمار در انغلستان انجام شده است، نتايج

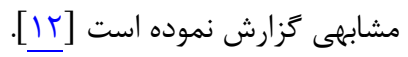

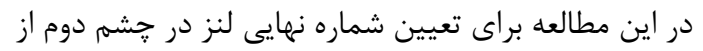

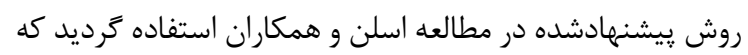

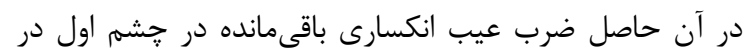

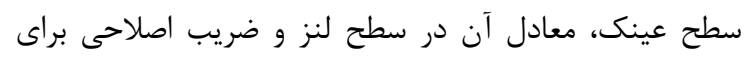

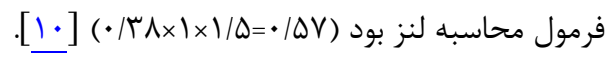

\section{نتيجه كيرى}

مطالعه حاضر با تعديل يارشيال قدرت لنز جشم دوم، نتايج

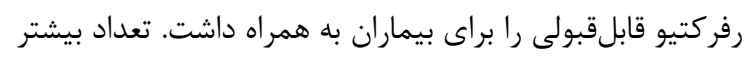

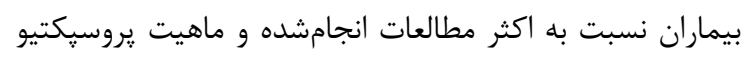

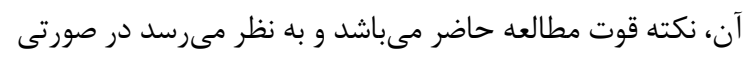

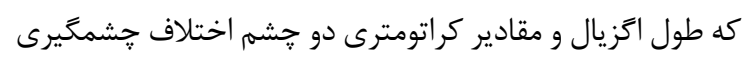

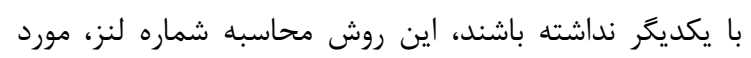

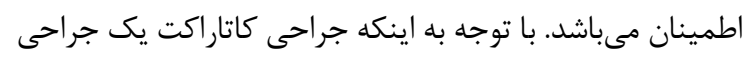

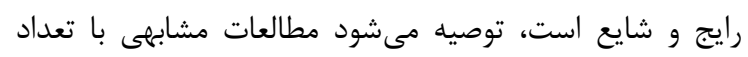

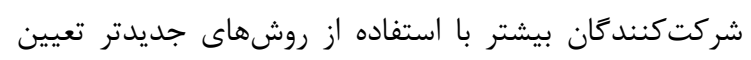

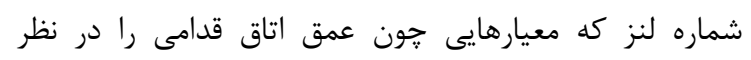

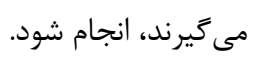

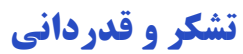

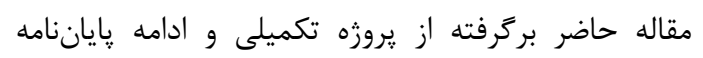

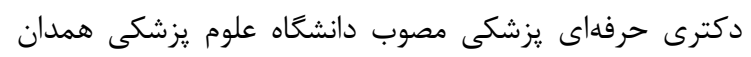

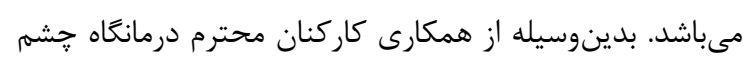

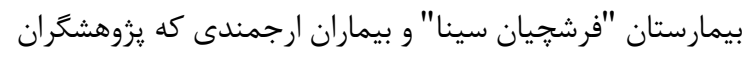

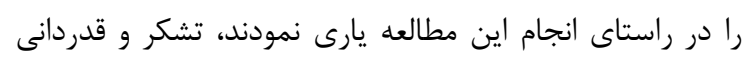
مى كردد.

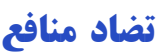
نتايج اين مطالعه با منافع نويسندكان در تعارض نمىباشد.

\section{ملاحظات اخلاقى}

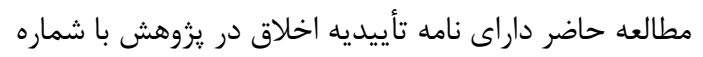

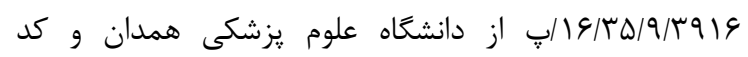

با بيشرفتهاى صورتكرفته در روش جراحى كاتاراكت،

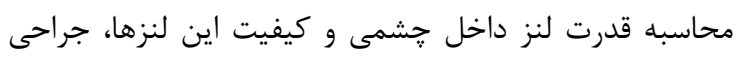

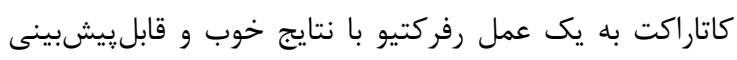

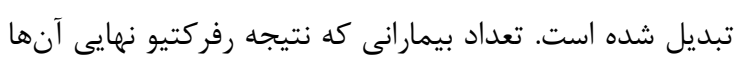

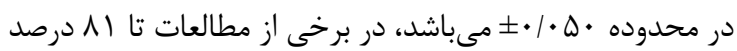

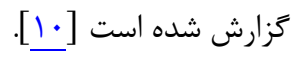

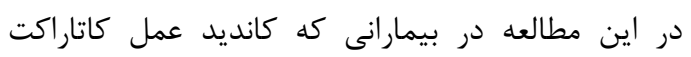

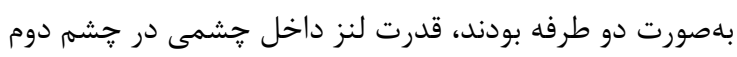

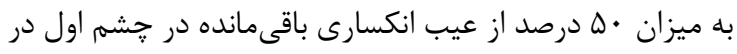

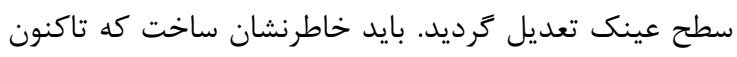

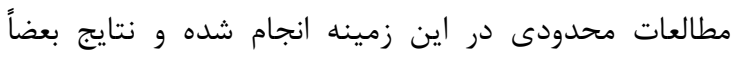

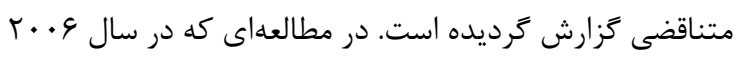

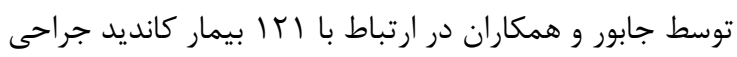

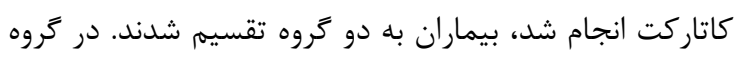

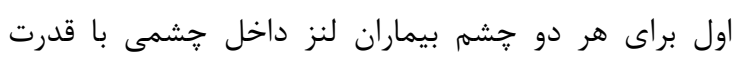

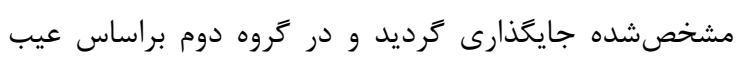

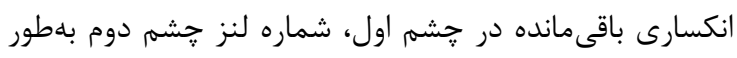

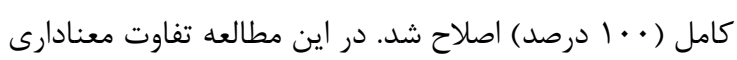

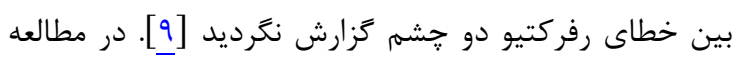

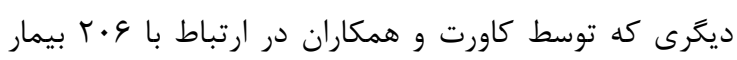

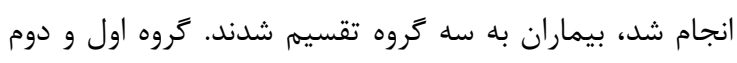

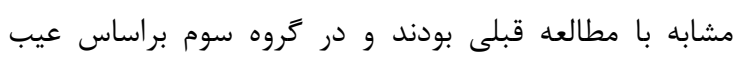

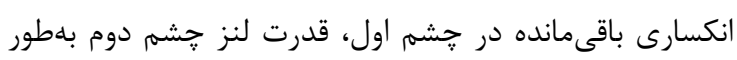

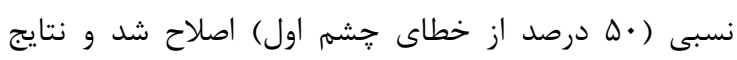

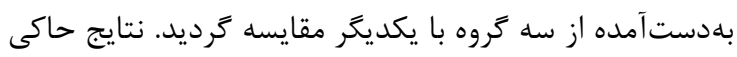

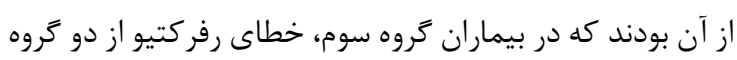

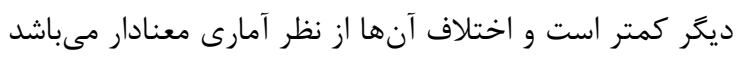

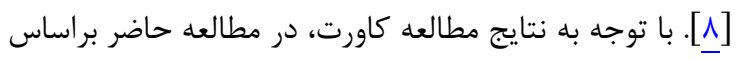

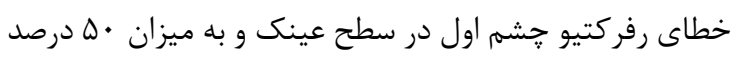

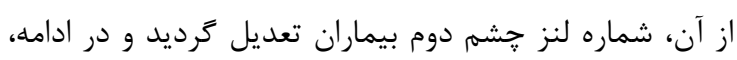

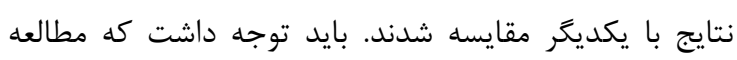

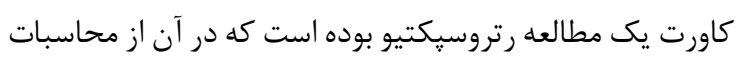

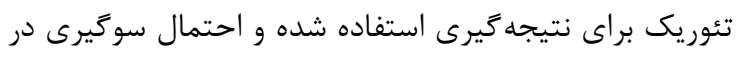

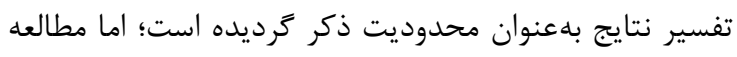

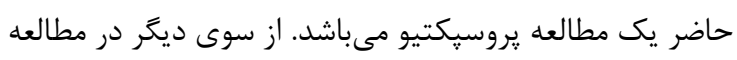

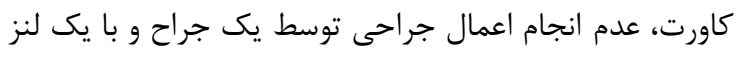

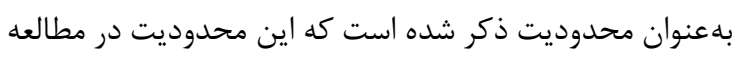

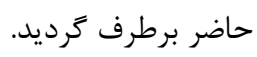

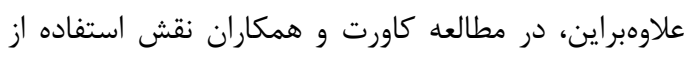

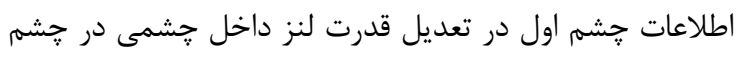
دوم تأييد شده است. در مطالعه حاضر نيز كه در آن برات نداى تعيين

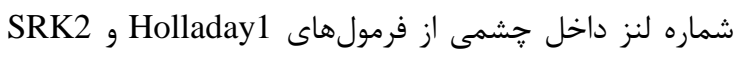

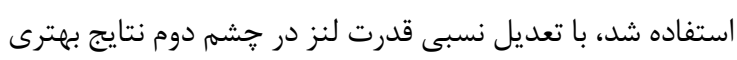




$$
\begin{aligned}
& \text { روششناسى و جمعآورى اطلاعات: · · درصد؛ نويسنده سوم }
\end{aligned}
$$

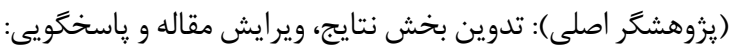

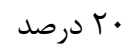$$
\text { حمايت مالى }
$$

\section{REFERENCES}

1. Murphy C, Tuft SJ, Minassian DC. Refractive error and visual outcome after cataract extraction. J Cataract Refract Surg. 2002; 28(1):62-6. PMID: 11777711 DOI: 10.1016/ s0886-3350(01)01027-6

2. Holladay JT. IOL power calculations for multifocal lenses. Texas: Cataract \& Refractive Surgery Today; 2007. P. 71-3

3. Behnding A, Montan P, Stenevi U, Kugelberg M, Lundstrom M. Aiming for emmetropia after cataract surgery: Swedish National Cataract Register study. J Cataract Refract Surg. 2012; 38(7): 1181-6. PMID: 22727287 DOI: 10.1016/j. jcrs.2012.02.035

4. Gökce SE, Zeiter JH, Weikert MP, Koch DD, Hill W, Wang L. Intraocular lens power calculations in short eyes. $J$ Cataract Refract Surg. 2017; 43(7):892-7. PMID: 28823434 DOI: $10.1016 /$ j.jcrs.2017.07.004

5. Mamalis N. Intraocular lens power accuracy. How are we doing? J Cataract Refract Surg. 2003; 29(1):1-3. PMID: 12551642 DOI: 10.1016/s0886-3350(02)02011-4

6. Olsen T. Improved accuracy of intraocular lens power calculation with the zeiss iol master. Acta Ophthalmol Scand. 2007; 85(1):84-7. PMID: 17244216 DOI: $10.1111 / \mathrm{j} .1600-$ 0420.2006.00774.x

7. Kugelberg M, Lundstrom M. Factors related to the degree of success in achieving target refraction in cataract surgery: Swedish national cataract register study. $J$ Cataract

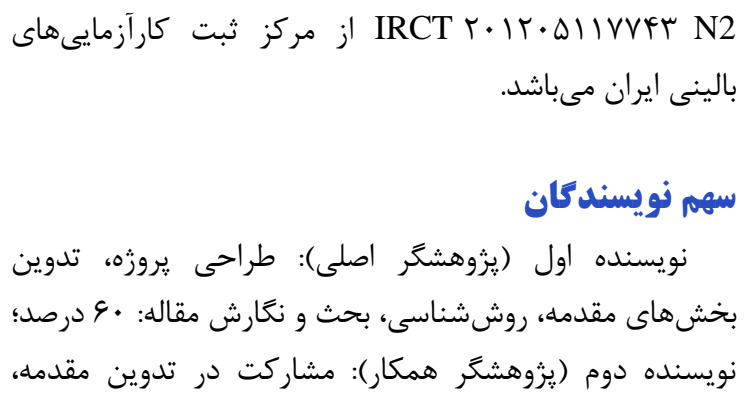

Refrect Surg. 2008; 34(11):1935-3. PMID: 19006741 DOI: 10.1016/j.jcrs.2008.06.036

8. Covert DJ, Henry CR, Koenig SB. Intraocular lens power selection in the second eye of patients undergoing bilateral sequential cataract extraction. Ophthalmology. 2010; 117(1): 49-54. PMID: 19815281 DOI: 10.1016/j.ophtha.2009.06.020

9. Jabbour J, Irwig L, Macaskill P, Hennessy MP. Intraocular lens power in bilateral cataract surgery: whether adjusting for error of predicted refraction in the first eye improves prediction in the second eye. J Cataract Refract Surg. 2006; 32(12):2091-7. PMID: 17137989 DOI: 10.1016/j.jcrs. 2006.08.030

10. Olsen T. Use of fellow eye data in the calculation of intraocular lens power for the second eye. Ophthalmol. 2011; 118(9):1710-5. PMID: 21723613 DOI: 10.1016/j.ophtha. 2011.04.030

11. Zhang J, Ning X, Yan Hong. Adjustment of IOL power for the second eye based on Refractive error of the first operated eye. Int J Ophthalmol. 2019; 12(8):1348-50. PMID: 31456928 DOI: $10.18240 /$ ijo.2019.08.18

12. Turnbull AMJ, Barrett GD. Using the first-eye prediction error in cataract surgery to refine the refractive outcome of the second eye. J Cataract Refract Surg. 2019;45(9):1239-45. PMID: 31326224 DOI: 10.1016/j.jcrs.2019.04.008 